\title{
Multi-band meta-material structures based on hexagonal shaped magnetic resonators
}

\author{
H. Benosman* and N. Boukli Hacene \\ Laboratory of Telecommunication, Faculty of Technology, University of Tlemcen, Algeria.
}

Accepted 15 April, 2013

\begin{abstract}
In this paper, we propose a new multi band meta-material structure composed of a unit cell that has multiple hexagonal-shaped metal rings printed one inside the other. The introduced meta-material composition exhibits multi-resonant behavior due to distinct magnetic resonances caused by the self and mutual coupling of each inclusion. The number of distinct magnetic resonances is determined by the number of hexagonal-shaped rings placed in the unit cell. By using three rings, three distinct resonance frequencies can be shifted to desired values simply by changing the design parameters what is described in the paper. Transmission and reflection spectra of the proposed structure are obtained by using the CST microwave studio to observe its resonance frequencies. Then, the effective permeability and effective permittivity are retrieved from the already computed complex S-parameters to demonstrate the presence of three distinct negative permeability bandwidths.
\end{abstract}

Key words: Left-handed material, hexagonal split ring resonator, negative permeability, simulation.

\section{INTRODUCTION}

There is much interest in meta-materials for the microwave and optical applications because their electromagnetic properties are vastly different from ordinary materials. A meta-material is a structural composite with unique electromagnetic properties due to the interaction of waves with the finer-scale periodicity of conventional materials (Semichaevsky and Akyurtlu, 2007). The person who discovered the concept of metamaterials is Veselago in (1968). He assumed an unknown material that has a negative permeability and permittivity in the same frequency range and it shows the abnormal of electromagnetic properties when the uniform plane-wave propagation was studied. As a result, Veselago referred to the materials as left-handed materials (LHM), which mean the electric field, magnetic field, and propagation vector are related by a left-handed rule which results in the phase and group velocities of an electromagnetic wave are anti-parallel. The first structure to prove the existence of meta-materials was a split ring resonator (SRR) structure that has a magnetic response without magnetic materials and it was introduced by Pendry et al. (1999). Magnetic properties of the SRR are considered as the substrate of the microstrip patch antenna instead of high permittivity materials because we can not only reduce the size of the patch, and moreover, we can improve the bandwidth of the antenna using high permeability materials for substrates of microstrip patch antennas (Mosallaei and Sarabandi, 2004). The SRR structure has a ring with a gap, which has nonzero the inductance and capacitance. The transmission coefficient of the SRRs is minimum at the magnetic resonance frequency, which is estimated from the inductance and capacitance. It is important to design SRR structures from the substrate of the microstrip components because the shape, the orientation and the arrangement of SRR structures change the inductance and capacitance This 


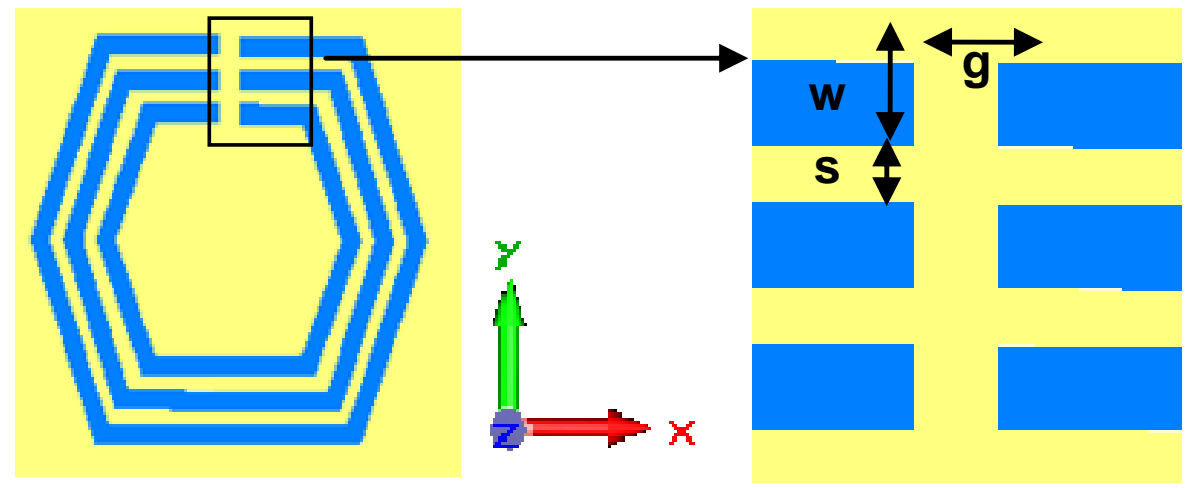

Figure 1. Front view of the hexagonal SRR unit cell.

means we can control and optimize the design of microstrip components by modifying structures in the substrate. There are several structures that have been introduced by the previous researchers. Ran et al. (2005) started with a symmetrical ring structure, then an omega, and finally an S structure. The SRR which has hexagonal cross section, different from the circular or square cross section of the SRRs in previous studies has been studied experimentally by Zhang et al. (2004) for X band. In the present paper a new three-ring SRR unit cell design is introduced for multi-band meta-material applications. For a given substrate material, design parameters are the side lengths and widths of metal strips, gap distances for each ring, and the separation distances between the rings. As a proof of concept, several multi-band SRR unit cells are designed and simulated in the paper for three different cases (for $N=1,2$ and 3 ). Complex $\mathrm{S}$ parameters transmission and reflection characteristics of the proposed hexagonal SRR (HSRR) are obtained by CST microwave studio, and are used for estimating the resonance frequencies of this novel structure or for the retrieval of the associated effective medium parameters $\varepsilon$ and $\mu$ (Smith and Schultz, 2002; Chen et al., 2004).

\section{DESIGN AND SIMULATIONS}

The structure suggested in this study is comprised of three hexagonal metallic rings maid of copper with conductivity of $5.8 \times 10^{7}$ $\mathrm{S} / \mathrm{m}$ and thickness of $0.035 \mathrm{~mm}$, laid out on the face of a dielectric substrate (R04003) with relative permittivity of 3.55. Dimensions of the substrate in the $x, y$ and $z$ directions are 8,8 and $0.81 \mathrm{~mm}$ respectively. The gap width $(\mathrm{g})$ and the width $(w)$ of each ring are both $0.33 \mathrm{~mm}$, while the separation distance between two adjacent copper lines $(s)$ is $0.1 \mathrm{~mm}$. Figure 1 represents the front view of the structure.

Meta-material unit cell is designed and simulated using the CST MWS software based on finite integration method. Two waveguide ports were set at the top and bottom of the X-axis, the substrate with multi-ring are centered in the waveguide and it is excited by an electromagnetic wave with the propagation vector $\mathrm{k}$ in $x$-direction, the electric field vector $\mathrm{E}$ in $y$-direction, and the magnetic field $\mathrm{H}$ in $z$-direction. Perfect electric conductor (PEC) boundary conditions were implemented on the left and the right of the $\mathrm{Y}$-axis, and perfect magnetic conductor (PMC) boundary conditions were placed in front and back of the Z-axis. Using this setup, single-ring, two-ring and three-ring SRR are designed and simulated.

Each one of the SRR structure simulated in the first step of this work are composed of only one type of single-ring hexagonalshaped unit cells for which one varied the side lengths (L). The lengths (L) are chosen to be 4, 3 and $2 \mathrm{~mm}$ for the SRR-A, SRR-B and SRR-C respectively as shown in Figure 2 . The same gap distance $(\mathrm{g})$ and the same metal strip width $(\mathrm{w})$ are used in these three different unit cells.

In the next step, a two-ring SRR unit cell is designed by combining the SRR-A and SRR-B unit cell topologies which are aligned in the gap-to-gap configuration as shown in Figure 3(a). Similarly, a three-ring SRR unit cell is also designed by combining the three types of unit cells SRR-A, SRR-B and SRR-C as illustrated in Figure 3(b).

To show the physical properties of the designed structures, $\mathrm{S}$ parameters for a single unit cell are calculated with the mentioned boundaries along the wave propagation. Next, the effective material parameters can be extracted from the $S$ parameters as described by Smith and Schultz (2002) and Chen et al. (2004).

$$
z=\sqrt{\frac{\left(1+S_{11}\right)^{2}-S_{21}^{2}}{\left(1-S_{11}\right)^{2}-S_{21}^{2}}} .
$$

$n=\frac{1}{k d} \cos ^{-1}\left[\frac{1}{2 S_{21}}\left(1-S_{11}{ }^{2}+S_{21}{ }^{2}\right)\right]$

Where $k$ and $d$ are the wave vector and the thickness of the slab. $z$ and $n$ indicate the wave impedance and refractive index, respectively. Then, the electric permittivity and magnetic permeability can be computed from the equations of $\varepsilon=n / z$ and $\mu=n z$.

\section{RESULTS}

The amplitude (in $\mathrm{dB}$ ) and the phase of $S_{11}$ and $S_{21}$ parameters (reflection-transmission coefficients) for the single-ring unit cell topologies SRR-A, SRR-B and SRR$C$ are shown in Figure 4. As it is seen, these parameters 


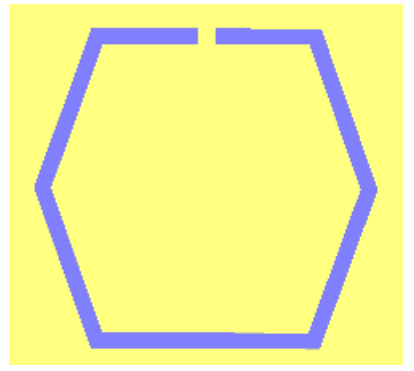

(a) SRR-A

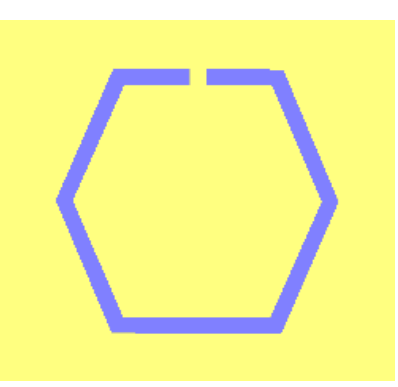

(b) SRR-B

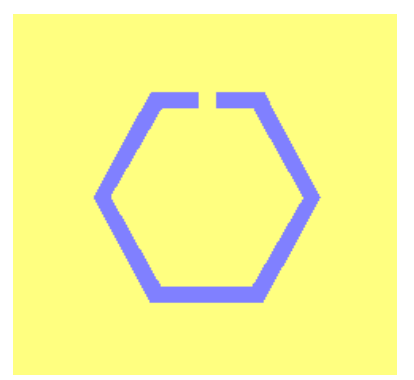

(c) SRR-C

Figure 2. Front view of the single-ring hexagonal-shaped SRR unit cells with different parameters.

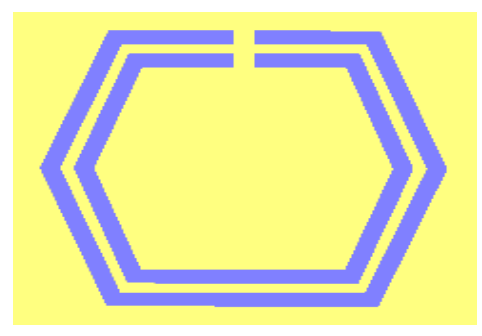

(a)

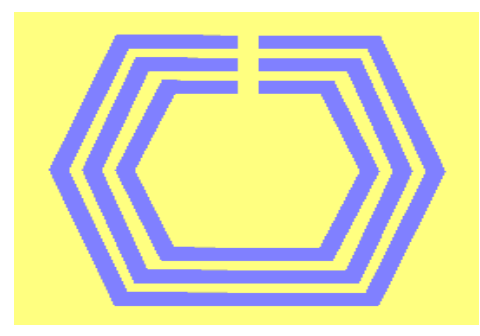

(b)

Figure 3. Multi-ring hexagonal SRR unit cell structures with two and three-rings.

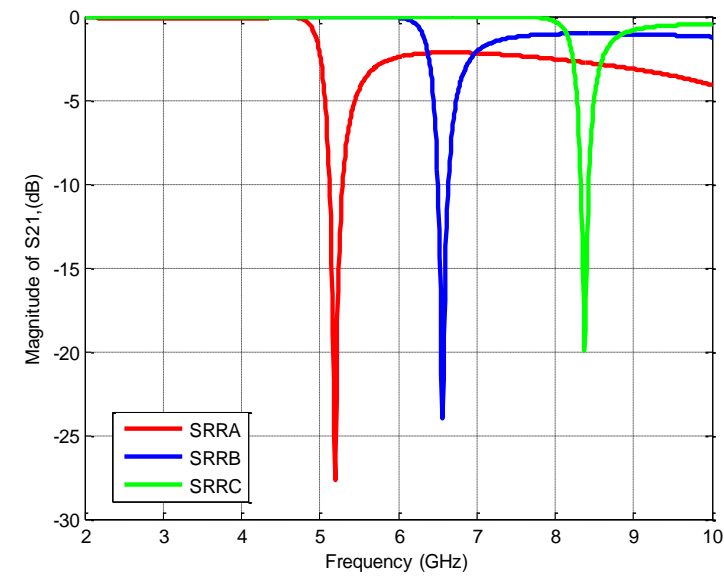

(a)

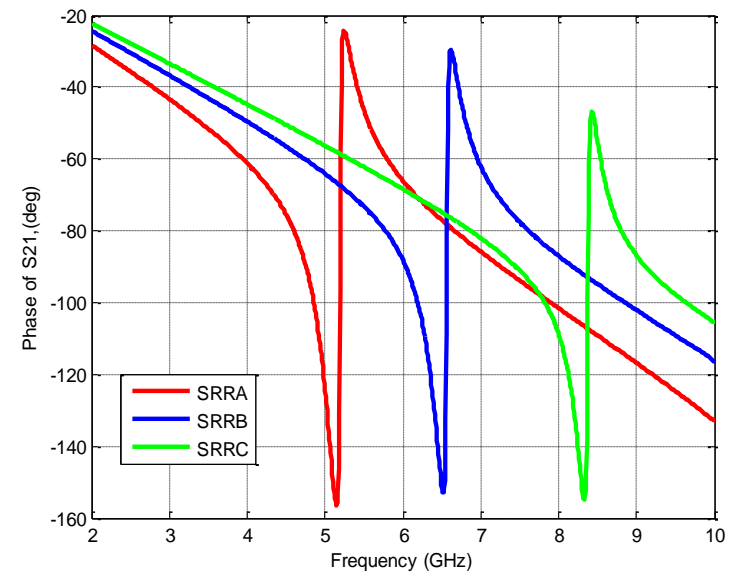

(b)

Figure 4. Magnitude and phase of $S_{21}$ for the single-ring hexagonal SRR.

are frequency dependent complex functions which satisfy certain requirements of causality. The dip in the phase of the transmission indicates the presence of negative region (or the location of the resonance in terms of the frequency) which can easily be observed from the figure. The simulation results are obtained for different values of side lengths (L) of the SRR metal rings 4,3 , and $2 \mathrm{~mm}$. The magnetic resonance frequencies associated occurs, respectively at $5.19,6.56$, and $8.37 \mathrm{GHz}$. As the side lengths decreases from 4 to $2 \mathrm{~mm}$, the minimum of the transmission and the dip of its phase shift from the lower to higher frequencies. It is obvious that one can easily 


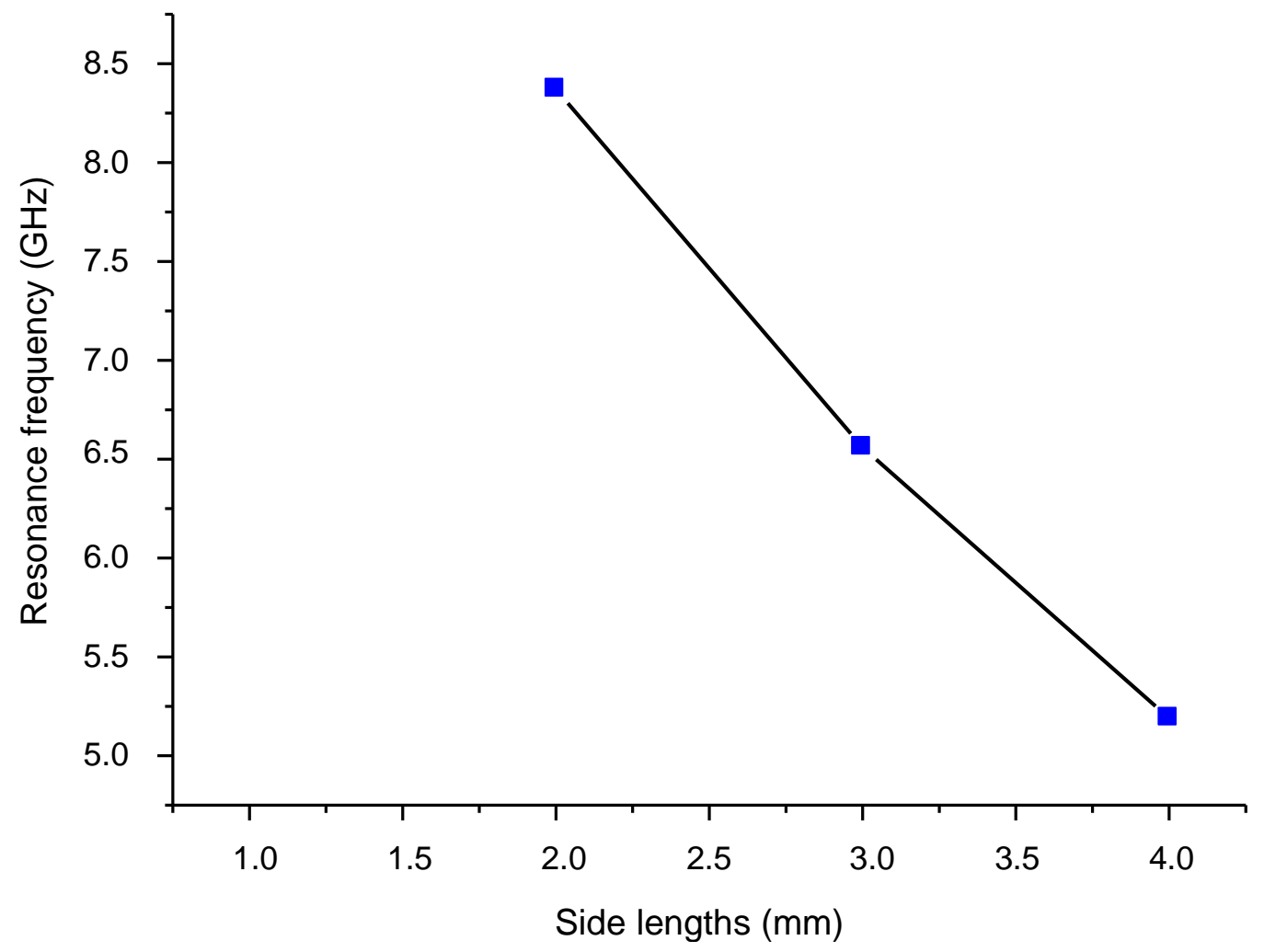

Figure 5. Dependence of resonance frequency on the side lengths.

Table 1. Resonance frequencies for the simulated SRR topologies.

\begin{tabular}{lcc}
\hline Structure & Resonance Freq 1. (GHz) square SRR & Resonance Freq 2. (GHz) hexagonal SRR \\
\hline SRR-A & 4.24 & 5.19 \\
SRR-B & 5.34 & 6.56 \\
SRR- C & 6.77 & 8.37 \\
two-ring SRR & $4.01,5.19$ & $5.08,6.28$ \\
three-ring SRR & $4.1,5.05,6.53$ & $5.06,6.17,8$ \\
\hline
\end{tabular}

obtain minimization or maximization in the frequency shifting as desired amount by tuning the side lengths. Thus, these kinds of structures can be considered as a resonant LC circuit with a resonance frequency which depends only of the inductance and capacitance of the structure $\omega=\frac{1}{\sqrt{L C}}$. When the side length is decreased, the capacitance will decrease, and thus the resonance frequency will increase as shown in Figure 5. Then, the minimum of the transmission and the dip of its phase will shift to the higher frequencies.

The magnitude and phase spectra of the $S_{21}$ and $S_{11}$ parameters are next computed for the two-ring SRR topology of Figure 3(a). Resulting plots are given in Figure 5 together with the plots for the real and imaginary parts of the effective permittivity, and effective permeability. It is clear from Figure 5 (a) that the structure resonates at two different frequencies, which are 5.08 and $6.28 \mathrm{GHz}$. Similarly Figure 5(b) shows the phase spectra of $S_{11}$ and $S_{21}$. The permeability is resonant in real part and takes negative values in the frequency range between 4.97 and $5.35 \mathrm{GHz}, 6.22$ and $6.4 \mathrm{GHz}$ as it appears in Figure 5(c), whereas, the real part of effective permittivity never goes below zero (Figure $5 \mathrm{~d}$ ). On the other hand, the three-ring SRR array has three distinct magnetic resonances at 5.06, 6.17 and $8 \mathrm{GHz}$ respectively. Figure 6(c) shows that this structure has three bands with negative real effective permeability, which are from 4.95 to $5.3 \mathrm{GHz}$, from 6.15 to $6.26 \mathrm{GHz}$ and from 7.98 to $8.03 \mathrm{GHz}$ (Figure 7a to d). Computed values of resonance frequencies of all hexagonal structures under investigation are summarized in Table 1. These results are in qualitative agreement with the results for square SRR presented by par Turkmen et al. 


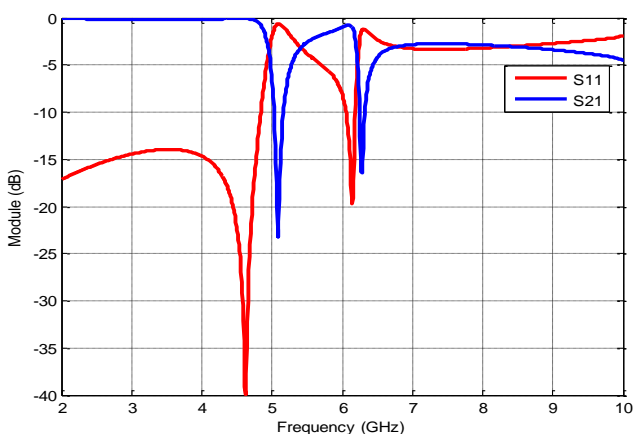

(a)

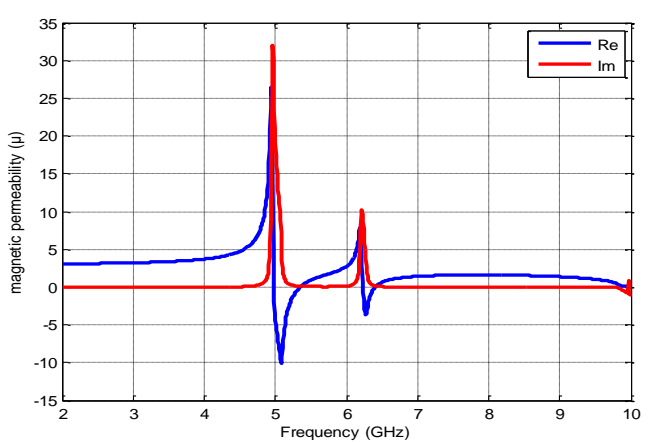

(c)

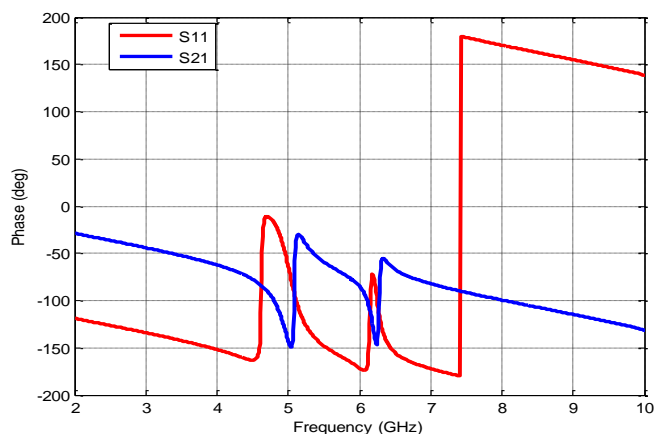

(b)

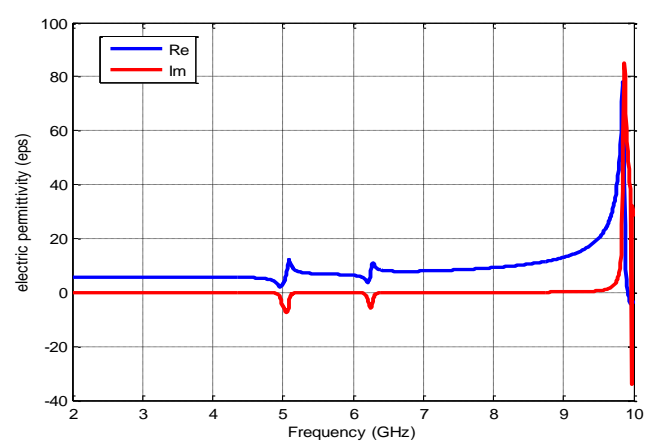

(d)

Figure 6. Results for the two-ring HSRR (a) Magnitude of $S_{11}$ and $S_{21}$, (b) Phase of $S_{11}$ and $S_{21}$. (c) Real and imaginary parts of the permeability, (d) Real and imaginary parts of the permittivity.

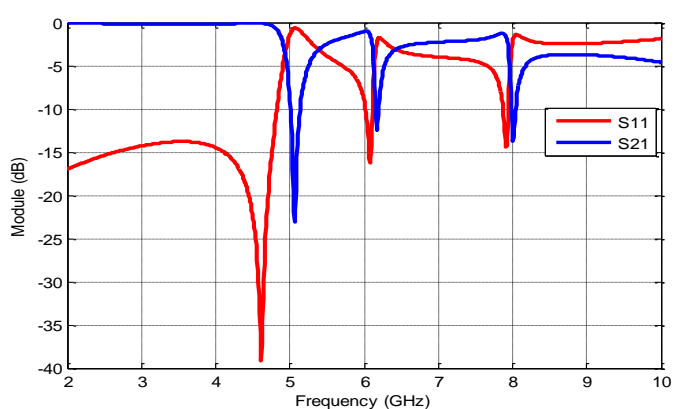

(a)

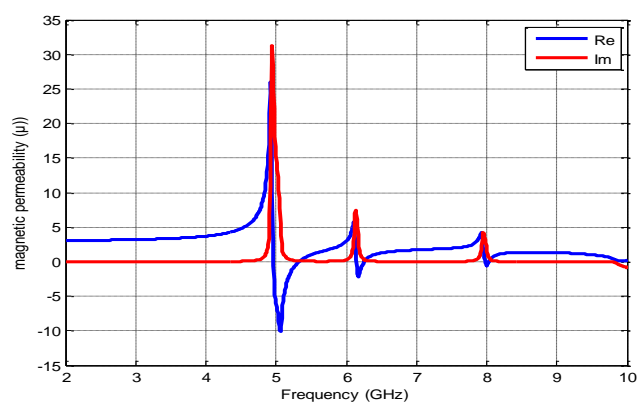

(c)

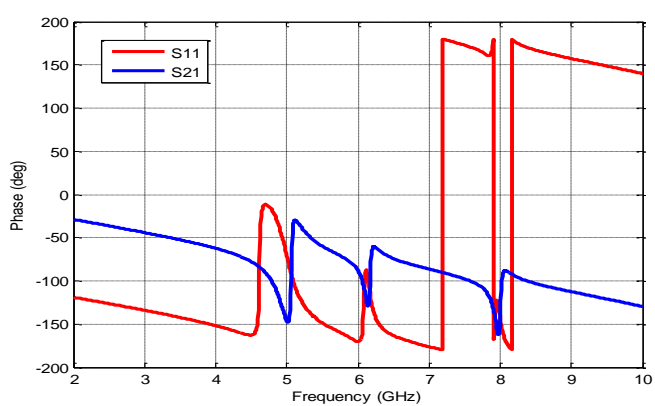

(b)

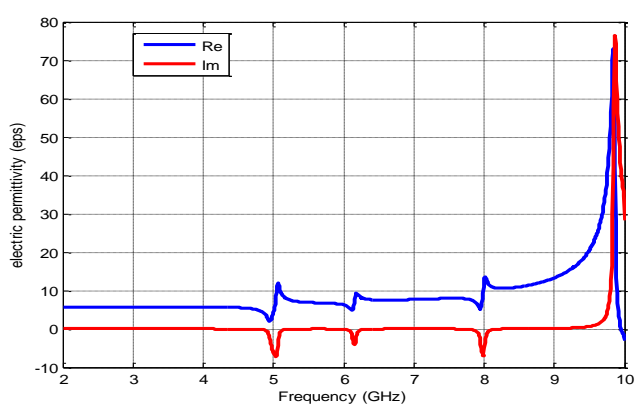

(d)

Figure 7. Results for the three-ring HSRR (a) Magnitude of $S_{11}$ and $S_{21}$, (b) Phase of $S_{11}$ and $S_{21}$. (c) Real and imaginary parts of the permeability, (d) Real and imaginary parts of the permittivity. 
(2011). It demonstrates that a desired number of magnetic resonances can be realized by selecting the number of concentric rings within the limits of geometrical constraints. It is also worth mentioning that resonance frequencies can also be adjusted by changing the design parameters $g, w$ and $s$. Thus, the interest of our structure suggested appears clearly in the miniaturization of the materials size used.

\section{Conclusion}

In this paper, three-ring hexagonal SRR structure for a new artificial magnetic inclusion has been introduced and the possibility of multiple magnetic resonances with negative permeability bands are demonstrated for these SRR topologies where the number of resonances is determined by the number of concentric rings. These results clearly show that the structure can be an alternative to the well known ordinary SRR structure to be used in the design of composite left-handed metamaterials especially when a multi-band magnetic resonator operation is needed. It is believed that the proposed multi-ring SRR design will provide an electrically small and easy-to-fabricate alternative to the present multi-band meta-material structures (Ekmekci et al., 2009; Ekmekci and Turhan-Sayan, 2010). The proposed resonant structure can be a good candidate for various meta-material applications in different portions of the electromagnetic spectrum, especially in the miniaturization of devices.

\section{REFERENCES}

Chen X, Grzegorczyk TM, Pacheco J, Kong JA (2004). Robust method to retrieve the constitutive effective parameters of metamaterials. Phys. Rev. E. 70:016608.1-016608.7.

Ekmekci E, Topalli K, Akin T, Turhan-Sayan G (2009). A tunable multiband metamaterial design using micro-split SRR structure. Opt Express. 17:16046-16058.
Ekmekci E, Turhan-Sayan G (2010). Single loop resonator: Dual-band magnetic metamaterial structure. Electron. Lett. 46:324-325.

Mosallae H, Sarabandi K (2004). Antenna miniaturization and bandwidth enhancement using reactive impedance substrate. IEEE transactions on antennas and propagation. 52:2403-2414.

Pendry J, Holden ADJ, Robbins DJWJ (1999). Stewart: Magnetism from conductors and enhanced nonlinear phenomena. IEEE Trans. Microwave Theory. Technol. 47:2075-2084.

Ran L, Huangfu J, Chen H, Zhang X, Cheng K, Grzegorczyk TM, Kong JA (2005). Experimental study on several left-handed metamaterials. Progress Electromagn. Res. 51:249-279.

Semichaevsky A, Akyurtlu A (2007). Homogenization of metamaterialloaded substrates and superstrates for antennas. Progress Electromagne. Res. 71:129-147.

Smith DR, Schultz S (2002). Determination of effective permittivity and permeability of metamaterials from reflection nd transmission coefficients. Phys. Rev. B. 65:195104.

Turkmen O, Ekmekci E, Turhan-Sayan G (2011). A new multi-ring SRR type meta-material design with multiple magnetic resonances. Progress in Electromagnetic Research Symposium, Marrakesh, Morocco, March, in press.

Veselago VG (1968). The electrodynamics of substances with simultaneously negative values of $\varepsilon$ and $\mu$. Sov. Phys. Uspekhi. 10:509-514.

Zhang F, Zhao Q, Liu Y, Luo C (2004). Behaviour of hexagon split ring resonators and left handed metamaterials. CHIN. PHIS LETT. 21:1330. 